\title{
Genetic Variations rs859, rs4646, and rs372883 in the 3'-Untranslated Regions of Genes Are Associated with a Risk of IgA Nephropathy
}

\author{
Xiaohong Yang ${ }^{a} \quad$ Gang Jin $^{\mathrm{b}}$ Yin Zhang ${ }^{\mathrm{a}}$ Maowei Xie ${ }^{\mathrm{a}}$ Wenning $\mathrm{Li}^{\mathrm{a}}$ \\ Daofa Zhang $^{\text {a }}$ Ru Wang ${ }^{a}$ Pei Zhang ${ }^{a}$ Yeguang Han ${ }^{a}$ Jiali Wei ${ }^{a}$ \\ aDepartment of Nephrology, Hainan General Hospital, Haikou, China; \\ ${ }^{\mathrm{b}}$ Renal Hemodialysis Center, Shaanxi Provincial People's Hospital, Xi'an, China
}

\section{Keywords}

IgA nephropathy · 3'-UTR · Polymorphism · Susceptibility

\begin{abstract}
Background: Previous studies indicate that genetic factors play an important role in the pathogenesis of IgA nephropathy (IgAN). To evaluate the association between single nucleotide polymorphisms (SNPs) in the 3'-untranslated region (3'-UTR) of genes and IgAN risk, we performed a case-control study in a Chinese Han population. Materials: Twelve SNPs were selected and genotyped in $384 \mathrm{IgAN}$ patients and 357 healthy controls. Odds ratio (OR) and 95\% confidence intervals $(\mathrm{Cl})$ were calculated by logistic regression adjusted for age and gender. Multifactor dimensionality reduction (MDR) was used to analyze the interaction of SNPSNP with IgAN risk. Results: Our study demonstrated that IL-16 rs859 (OR $=0.75, p=0.040)$ and CYP19A1 rs4646 (OR $=2.58, p=0.017)$ polymorphism were related to the risk of IgAN. In stratified analyses by gender, CYP19A1 rs4646 (OR $=2.96, p=0.015)$ and BACH1 rs372883 $(\mathrm{OR}=1.81, p=0.038)$ polymorphisms conferred susceptibility to IgAN in males. Besides, rs372883 reduced IgAN risk in females $(O R=0.44, p=0.042)$. We also found rs859 polymorphism was correlated with grade I-II (OR $=0.42, p=0.028)$ in subgroup analysis of Lee's clas-
\end{abstract}




\section{Kidney \\ Blood Pressure \\ Research}

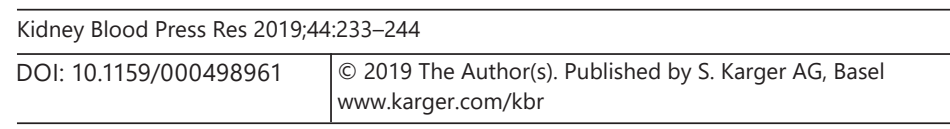

Yang et al.: Genetic Variations rs859, rs4646, and rs372883 Are Associated with IgA Nephropathy Risk

sification. Additionally, we found rs 4646 polymorphism was correlated with serum creatinine ( $p=0.035$ ). Conclusion: Our results suggested that the IL-16 rs859, CYP19A1 rs4646, and $B A C H 1$ rs372883 polymorphisms have potential roles in the genetic susceptibility to IgAN in Chinese Han population.

\section{Introduction}

Immunoglobulin A nephropathy (IgAN), also known as Berger's disease, is the most common type of primary glomerulonephritis worldwide [1]. IgAN also is the main cause of chronic kidney disease, and approximately $40 \%$ of IgAN patients progress to end-stage renal disease (ESRD) following diagnosis [2]. The prevalence of IgAN is particularly high in individuals of Asian ancestry, but is rare among Africans and is of intermediate prevalence among Europeans $[3,4]$. The disease is characterized by the presence of IgA-dominant or co-dominant immune deposits in the glomeruli, accompanied by the proliferation of mesangial cells and expansion of matrix [5]. Clinical manifestations of IgAN are usually macroscopic hematuria and different degrees of proteinuria [6]. Some clinical parameters are strong indicators of severe disease progression, such as increased serum creatinine concentration, hypercholesterolaemia, lower serum albumin level, severe proteinuria and the activation of complement (mainly C3 or C4) [7, 8]. Although research on IgAN has been ongoing for decades, the pathogenesis of IgAN remains poorly understood. It has been widely accepted that the genetic and environmental factors play vital roles in the etiology of IgAN, especially genetic factors.

Single nucleotide polymorphisms (SNPs)are the most common type of genetic variation in the human genome, which may help predict individual susceptibility to various diseases or understand the contribution of genetic factors to disease development [9-11]. SNPs located in the 3'-UTRs of genes may affect the expression of the gene by reinforcing, weakening or disrupting the miRNA-mRNA interaction, and thereby confer the individual's disease risk [12]. Thus, it gives us a clue that SNPs in the 3 '-UTR of genes can be candidate biomarkers, which may be a valuable indication and provide accurate prediction of diseases risk and progression. However, very few studies have been performed to investigate the role of these SNPs in the risk of IgAN.

To investigate the effect of genetic variants in the 3'-UTR of targeted genes (FBN1, CYP19A1, IL16, C16orf72, NTN1, CYP24A1, NRIP1, BACH1, TMPRSS2 and MTMR3) on the development of IgAN, we performed a case-control study to assess the possible effect of these 3 '-UTR polymorphisms on IgAN risk in a Chinese Han population.

\section{Materials and Methods}

\section{Subjects}

A total of unrelated 741 subjects were enrolled from the First Affiliated Hospital of Xi'an Jiaotong University, including 357 patients with renal biopsy verified IgAN and 384 geographically ethnicity-matched healthy controls. All patients were confirmed by histopathological examination of renal biopsy. The healthy controls were recruited from routine health examination in the same hospitals during the same period. Patients were excluded as followings: (1) systemic diseases, for example, systemic lupus erythematosus (SLE), diabetes and chronic liver disease; (2) Henoch-Schönlein purpura nephritis, secondary IgAN, acute interstitial nephritis and other renal diseases, such as diabetic nephropathy, hypertensive nephropathy; 
Kidney
Blood Pressure

Research

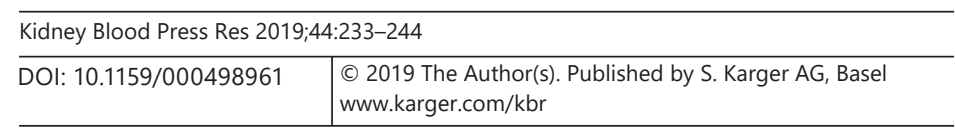

Yang et al.: Genetic Variations rs859, rs4646, and rs372883 Are Associated with IgA Nephropathy Risk

(3) patients with renal transplantation or malignancy. Healthy individuals without history of renal disease or chronic disease, such as diabetes, hypertension, or aggressive metabolic and endocrinological disease were recruited as controls. The detailed demographic and clinical data were collected from medical records and a structured questionnaire by interviewers.

The study was approved by the ethics committee of the First Affiliated Hospital of Xi'an Jiaotong University, and the experimental protocol was implemented in accordance with the Declaration of Helsinki. Written informed consents were obtained from all participants before taking part in this research.

\section{SNP Selections}

This study focused on the relationship between SNPs in the 3 '-UTR of the genes, which were associated with some immunological diseases and IgAN risk in Chinese Han population. The $3^{\prime}$-UTR sequences of these candidate genes were identified using the NCBI nucleotide database (http://www.ncbi.nlm.nih/gov). Information regarding genetic variations of these 3'-UTR SNP was obtained by an extensive search in the NCBI database (dbSNP, http://www.ncbi.nlm.nih. gov/projects/SNP/) and the UCSC genome browser (http://genome.ucsc.edu/). Finally, twelve SNPs were chosen for analysis in our study, all of which had minor allele frequencies (MAFs) $>5 \%$ in the 1000 Genomes Project data (http://www.internationalgenome.org/). Online software miRbase (http://www.mirbase.org/), and TargetScan 7.1 (http://www.targetscan. org) were used to predict the miRNAs binding within $3^{\prime}$-UTR of these genes.

\section{DNA Extraction and Genotyping}

Five milliliters of peripheral blood samples were collected into tubes containing the anticoagulant EDTA and stored at $-80^{\circ} \mathrm{C}$ for later analysis. Genomic DNA was extracted using the GoldMag DNA Purification Kit (GoldMag Co. Ltd, Xi'an City, China) according to the manufacturer's instructions, and the purity and concentration of DNA were measured by the NanoDrop2000 (Thermo Scientifc, Waltham, MA, USA).

Primers for amplification and single base extension were designed using the MassARRAY Assay Design v3.0 software (Agena, San Diego, CA, USA) according to the standard protocol recommended by the manufacturer. These SNPs were genotyped by the Agena MassARRAY system by two laboratory personnel independently in a double-blinded fashion. Agena Typer 4.0 software was used for data management and analysis. For quality control, approximately $10 \%$ of samples were randomly selected to repeat genotyping, and the reproducibility was $100 \%$.

\section{Statistical Analysis}

Statistical analysis was performed with SPSS 18.0 (SPSS, Chicago, IL, USA). The independent-samples $t$ tests (for continuous variables) or the $\chi^{2}$ test $\left(\chi^{2}\right.$ test, for categorical variables) was used to examine the differences in the distribution of demographic characteristics between patients and controls. SNPs frequencies in the control subjects were tested for departure from Hardy-Weinberg Equilibrium (HWE) by the Fisher's exact test. SNP allele and genotype frequencies of cases and controls were compared by a $\chi^{2}$ test. Associations between these SNPs and IgAN risk were evaluated by calculating odds ratios (ORs) and 95\% confidence intervals (CIs) using logistic regression analysis after adjusting for age and gender. The wild-type allele was used as a reference. Multiple inheritance models (codominant, dominant, recessive and log-additive) were estimated by SNPstats online tools software (http://bioinfo. iconcologia.net/snpstats/start.htm). Multifactor dimensionality reduction (MDR) (version 3.0.2) was performed to evaluate the SNP-SNP interactions in the risk of IgAN. The difference in data for clinical characteristics among different genotypes was analyzed using the one-way analysis and ANOVA test. All tests were two-sided and $p$ value $<0.05$ was considered as statistical significance. 
Table 1. Characteristics of patients with IgA nephropathy and controls

\begin{tabular}{lccc}
\hline Characteristics & Cases $(n=357)$ & Controls $(n=384)$ & $p$ \\
\hline Age, years & & & $<0.001$ \\
$\quad$ Mean \pm SD & $32.44 \pm 11.80$ & $51.16 \pm 11.49$ & 0.036 \\
Gender & & & \\
$\quad$ Male & $233(65.26 \%)$ & $278(72.40 \%)$ & $<0.001$ \\
$\quad$ Female & $124(34.74 \%)$ & $106(27.60 \%)$ & 0.612 \\
Casts, $n / \mu \mathrm{L}$ & $5.82 \pm 20.17$ & $0.43 \pm 0.65$ & 0.138 \\
Urine protein, g/day & $2.73 \pm 6.37$ & $33.02 \pm 7.29$ & \\
ALB, g/L & $33.98 \pm 7.98$ & $4.55 \pm 3.83$ & \\
CHO, mmol/L & $6.11 \pm 18.60$ & & $<0.001$ \\
Serum C3, g/L & $1.06 \pm 0.26$ & & $<0.001$ \\
Serum C4, g/L & $0.27 \pm 0.10$ & $67.88 \pm 53.55$ & \\
BUN, mmol/L & $8.20 \pm 5.92$ & $335.81 \pm 100.03$ & \\
SCr, $\mu$ mol/L & $145.70 \pm 159.79$ & & \\
UA, $\mu$ mol/L & $380.09 \pm 192.03$ & & \\
Serum $\beta$ macroglobulin, $\mu \mathrm{g} / \mathrm{L}$ & $2,980.23 \pm 2,408.85$ & & \\
Hemoglobin, g/L & $127.25 \pm 23.97$ & & \\
Lee's classification & & & \\
$\quad$ I-II & 120 & & \\
$\quad$ III-V & 214 & & \\
$\quad$ Missing & 22 & & \\
\hline
\end{tabular}

SD, standard deviation; $\mathrm{ALB}$, serum albumin; $\mathrm{CHO}$, serum cholesterol; $\mathrm{C} 3$, complement 3; C4, complement 4; BUN, blood urea nitrogen; SCr, serum creatinine; UA, serum uric acid.

\section{Results}

The study included 357 patients with IgAN (233 males and 124 females) and 384 healthy controls (278 males and 106 females). The age of the cases and the controls was $32.44 \pm 11.8$ years and $51.16 \pm 11.49$ years, respectively. Demographic and clinical characteristics were shown in Table 1, including age, gender, casts, $24 \mathrm{~h}$ urine protein, serum albumin level (ALB), serum cholesterol level (CHO), serum C3 level, serum C4 level, blood urea nitrogen (BUN), serum creatinine level (SCr), serum uric acid (UA), Serum $\beta$ macroglobulin and hemoglobin.

Totally, twelve 3 '-UTR SNPs were successfully genotyped, and the average variant call rate was $99.33 \%$. Details of candidate SNPs were listed in Table 2. All SNPs were in HWE in the controls $(p>0.05)$ except for rs462574, which was excluded from subsequent analyses. The MAFs of all SNPs were greater than $5 \%$ in the study population. Differences in SNP allele frequency distribution between the IgAN patients and the healthy controls were analyzed using $\chi^{2}$ tests and odds ratios (ORs), although no statistically significant difference was observed (all $p>0.05$ ) (Table 2).

We tested the association between SNPs and IgAN risk under different inheritance models (codominant, dominant, recessive and log-additive) by using logistic regression adjusted for age and gender. As shown in Table 3, we observed that $I L-16$ rs859 and CYP19A1 rs4646 polymorphisms were associated with the susceptibility of IgAN. Rs859 polymorphism was associated with a decreased risk of IgAN under the log-additive model (OR $=0.75,95 \%$ $\mathrm{CI}=0.57-0.99, p=0.040$ ). In contrast, individuals carrying the rs4646 AA genotype had a higher risk of IgAN in the codominant model (OR $=2.38,95 \% \mathrm{CI}=1.08-5.27, p=0.036)$ and recessive model $(\mathrm{OR}=2.58,95 \% \mathrm{CI}=1.19-5.61, p=0.017)$, respectively. However, significant association between other SNPs and IgAN susceptibility was not observed. 
Table 2. Allele frequencies in cases and controls and odds ratio estimates for IgA nephropathy risk

\begin{tabular}{|c|c|c|c|c|c|c|c|c|c|c|}
\hline \multirow[t]{2}{*}{ SNP ID } & \multirow[t]{2}{*}{ Gene } & \multirow{2}{*}{$\begin{array}{l}\text { Loca- } \\
\text { tion }\end{array}$} & \multirow[t]{2}{*}{ Position } & \multirow[t]{2}{*}{ Role } & \multirow{2}{*}{$\begin{array}{l}\text { Alleles } \\
\text { (A/B) }\end{array}$} & \multicolumn{2}{|l|}{ MAF } & \multirow{2}{*}{$\begin{array}{l}p \text { value } \\
\text { for HWE }\end{array}$} & \multirow[t]{2}{*}{ OR $(95 \% \mathrm{CI})$} & \multirow[t]{2}{*}{$p$ value } \\
\hline & & & & & & cases & controls & & & \\
\hline rs1042078 & FBN1 & 15 & 48702873 & UTR-3 & $\mathrm{G} / \mathrm{A}$ & 0.448 & 0.454 & 0.607 & $0.97(0.79-1.20)$ & 0.796 \\
\hline rs2255192 & CYP19A1 & 15 & 51500835 & UTR-3 & $\mathrm{T} / \mathrm{C}$ & 0.185 & 0.178 & 0.491 & $1.05(0.80-1.37)$ & 0.727 \\
\hline rs4646 & CYP19A1 & 15 & 51502844 & UTR-3 & $\mathrm{A} / \mathrm{C}$ & 0.279 & 0.260 & 0.691 & 1.10 (0.88-1.39) & 0.409 \\
\hline rs859 & IL16 & 15 & 81601322 & UTR-3 & $\mathrm{A} / \mathrm{G}$ & 0.475 & 0.487 & 0.839 & $0.95(0.78-1.17)$ & 0.637 \\
\hline rs1048687 & C16orf72 & 16 & 9212987 & UTR-3 & $\mathrm{T} / \mathrm{C}$ & 0.459 & 0.474 & 0.919 & $0.94(0.77-1.16)$ & 0.571 \\
\hline rs9889875 & NTN1 & 17 & 9147259 & UTR-3 & $\mathrm{C} / \mathrm{T}$ & 0.490 & 0.451 & 0.303 & $1.17(0.96-1.44)$ & 0.126 \\
\hline rs4809957 & CYP24A1 & 20 & 52771171 & UTR-3 & $\mathrm{A} / \mathrm{G}$ & 0.412 & 0.387 & 0.830 & $1.11(0.90-1.37)$ & 0.324 \\
\hline rs1056930 & NRIP1 & 21 & 16336804 & UTR-3 & $\mathrm{T} / \mathrm{C}$ & 0.053 & 0.066 & 1.000 & $0.79(0.51-1.22)$ & 0.286 \\
\hline rs372883 & BACH1 & 21 & 30717737 & UTR-3 & $\mathrm{C} / \mathrm{T}$ & 0.449 & 0.436 & 0.835 & $1.06(0.86-1.30)$ & 0.604 \\
\hline rs 462574 & TMPRSS2 & 21 & 42836729 & UTR-3 & $\mathrm{G} / \mathrm{A}$ & 0.490 & 0.474 & $0.010^{\#}$ & - & - \\
\hline rs456298 & TMPRSS2 & 21 & 42836751 & UTR-3 & $\mathrm{A} / \mathrm{T}$ & 0.452 & 0.439 & 0.408 & $1.06(0.86-1.30)$ & 0.599 \\
\hline rs12537 & MTMR3 & 22 & 30423460 & UTR-3 & $\mathrm{T} / \mathrm{C}$ & 0.207 & 0.229 & 1.000 & $0.88(0.69-1.13)$ & 0.308 \\
\hline
\end{tabular}

SNP, single nucleotide polymorphism; MAF, minor allele frequency; HWE, Hardy-Weinberg equilibrium. $p$ values were calculated with Pearson's $\chi^{2}$ tests; $p<0.05$ indicates statistical significance. ${ }^{\#} p$ value for HWE was $<0.05$, which was significantly deviated from the HWE and excluded from subsequent analyses.

Table 3. Relationships between IL16 and CYP19A1 polymorphism and IgA nephropathy risk adjusted by gender and age

\begin{tabular}{|c|c|c|c|c|c|c|c|c|c|}
\hline Gene & SNP ID & Model & Genotype & Control & Case & OR $(95 \% \mathrm{CI})$ & $p$ & AIC & $\mathrm{BIC}$ \\
\hline \multirow[t]{8}{*}{ IL16 } & \multirow[t]{8}{*}{ rs859 } & \multirow[t]{3}{*}{ Codominant } & $\mathrm{G} / \mathrm{G}$ & $102(26.6 \%)$ & $97(27.2 \%)$ & 1.00 & \multirow{3}{*}{0.120} & \multirow{3}{*}{669.9} & \multirow{3}{*}{693.0} \\
\hline & & & $\mathrm{G} / \mathrm{A}$ & $190(49.5 \%)$ & $180(50.6 \%)$ & $0.71(0.44-1.12)$ & & & \\
\hline & & & $\mathrm{A} / \mathrm{A}$ & $92(24 \%)$ & $79(22.2 \%)$ & $0.57(0.33-0.98)$ & & & \\
\hline & & \multirow[t]{2}{*}{ Dominant } & $\mathrm{G} / \mathrm{G}$ & $102(26.6 \%)$ & $97(27.2 \%)$ & 1.00 & \multirow{2}{*}{0.062} & \multirow{2}{*}{668.8} & \multirow{2}{*}{687.2} \\
\hline & & & $\mathrm{G} / \mathrm{A}-\mathrm{A} / \mathrm{A}$ & $282(73.4 \%)$ & $259(72.8 \%)$ & $0.66(0.42-1.02)$ & & & \\
\hline & & \multirow[t]{2}{*}{ Recessive } & $\mathrm{G} / \mathrm{G}-\mathrm{G} / \mathrm{A}$ & $292(76 \%)$ & 277 (77.8\%) & 1.00 & \multirow{2}{*}{0.140} & \multirow{2}{*}{670.1} & \multirow{2}{*}{688.5} \\
\hline & & & $\mathrm{A} / \mathrm{A}$ & $92(24 \%)$ & $79(22.2 \%)$ & $0.72(0.46-1.12)$ & & & \\
\hline & & Log-additive & - & - & - & $0.75(0.57-0.99)$ & $0.040^{*}$ & 668.1 & 686.5 \\
\hline \multirow[t]{8}{*}{ CYP19A1 } & \multirow[t]{8}{*}{ rs4646 } & Codominant & $\mathrm{C} / \mathrm{C}$ & $208(54.2 \%)$ & 187 (52.5\%) & 1.00 & \multirow{3}{*}{$0.036^{*}$} & \multirow{3}{*}{668.7} & \multirow{3}{*}{691.7} \\
\hline & & & $\mathrm{C} / \mathrm{A}$ & $152(39.6 \%)$ & $139(39 \%)$ & $0.82(0.55-1.23)$ & & & \\
\hline & & & $\mathrm{A} / \mathrm{A}$ & $24(6.2 \%)$ & $30(8.4 \%)$ & 2.38 (1.08-5.27) & & & \\
\hline & & \multirow[t]{2}{*}{ Dominant } & $\mathrm{C} / \mathrm{C}$ & $208(54.2 \%)$ & 187 (52.5\%) & 1.00 & \multirow{2}{*}{0.810} & \multirow{2}{*}{673.3} & \multirow{2}{*}{691.7} \\
\hline & & & $\mathrm{C} / \mathrm{A}-\mathrm{A} / \mathrm{A}$ & $176(45.8 \%)$ & $169(47.5 \%)$ & $0.95(0.65-1.39)$ & & & \\
\hline & & \multirow[t]{2}{*}{ Recessive } & $\mathrm{C} / \mathrm{C}-\mathrm{C} / \mathrm{A}$ & $360(93.8 \%)$ & $326(91.6 \%)$ & 1.00 & \multirow{2}{*}{$0.017^{*}$} & \multirow{2}{*}{667.6} & \multirow{2}{*}{686.1} \\
\hline & & & $\mathrm{A} / \mathrm{A}$ & $24(6.2 \%)$ & $30(8.4 \%)$ & $2.58(1.19-5.61)$ & & & \\
\hline & & Log-additive & - & - & - & $1.13(0.83-1.53)$ & 0.450 & 672.8 & 691.2 \\
\hline
\end{tabular}

OR, odds ratio; 95\% CI, 95\% confidence interval; AIC, Akaike information criterion; BIC, Bayesian information criterion. $p$ values were calculated by unconditional logistic regression analysis with adjustments for age and gender. ${ }^{*} p<0.05$ means the data is statistically significant. 
Kidney

Blood Pressure

Research
Kidney Blood Press Res 2019;44:233-244 (c) 2019 The Author(s). Published by S. Karger AG, Basel www.karger.com/kbr

Yang et al.: Genetic Variations rs859, rs4646, and rs372883 Are Associated with IgA Nephropathy Risk

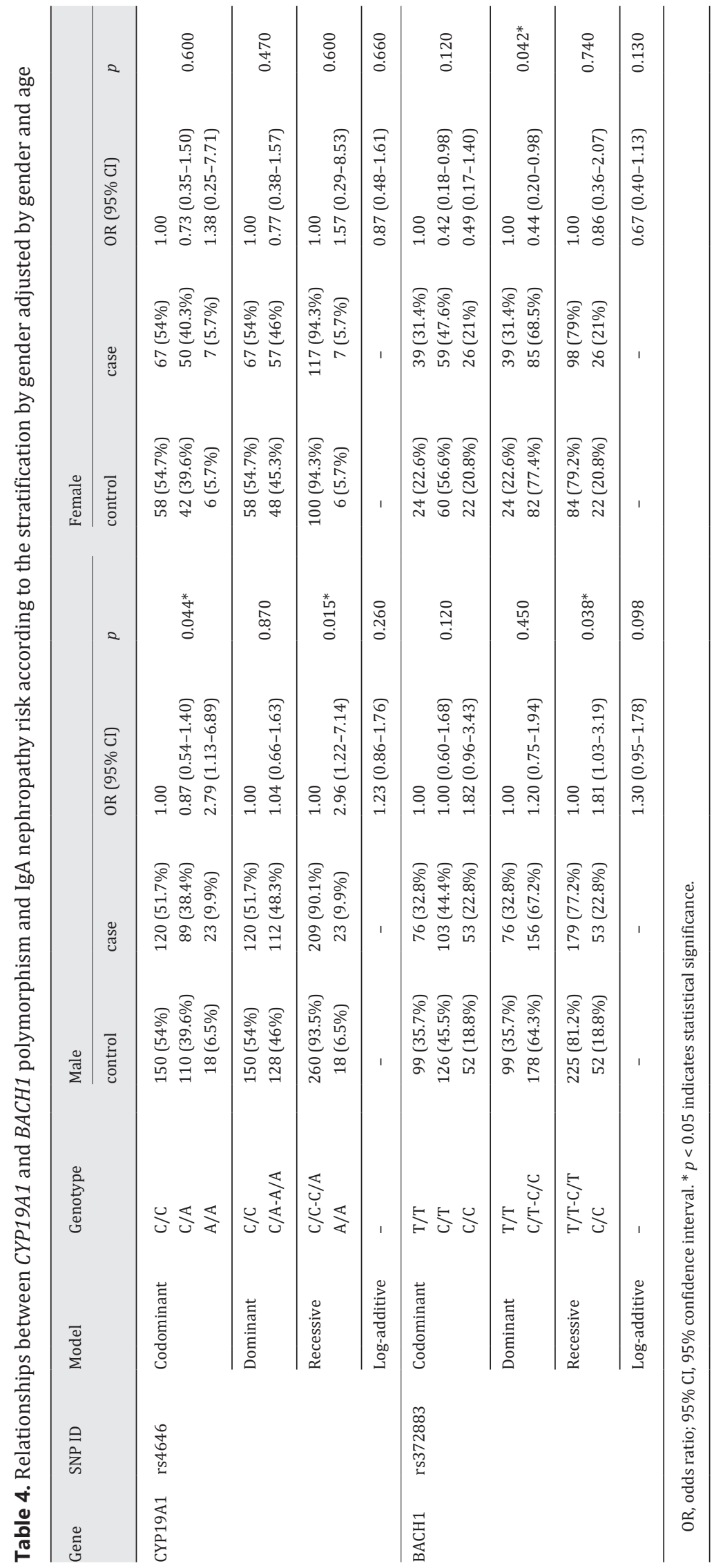


Yang et al.: Genetic Variations rs859, rs4646, and rs372883 Are Associated with IgA Nephropathy Risk

Table 5. Subgroup analysis by Lee's classification for the relationships between IL16 rs859 and IgA nephropathy risk

\begin{tabular}{|c|c|c|c|c|c|c|c|c|}
\hline \multirow[t]{2}{*}{ Model } & \multirow[t]{2}{*}{ Genotype } & \multirow[t]{2}{*}{ Controls, $n(\%)$} & \multicolumn{3}{|l|}{ Grade I-II } & \multicolumn{3}{|l|}{ Grade III-V } \\
\hline & & & case, $n(\%)$ & OR (95\% CI) & $p$ & case, $n(\%)$ & OR (95\% CI) & $p$ \\
\hline \multirow[t]{2}{*}{ Allele } & $\mathrm{G}$ & $394(51.3 \%)$ & $128(53.3 \%)$ & 1.00 & \multirow{2}{*}{0.605} & $222(51.9 \%)$ & 1.00 & \multirow{2}{*}{0.857} \\
\hline & A & $374(48.7 \%)$ & $112(46.7 \%)$ & $0.92(0.69-1.23)$ & & $206(48.1 \%)$ & $0.98(0.77-1.24)$ & \\
\hline \multirow[t]{3}{*}{ Genotype } & $\mathrm{G} / \mathrm{G}$ & $102(26.6 \%)$ & $35(29.2 \%)$ & 1.00 & \multirow{3}{*}{$\begin{array}{l}0.055 \\
0.028^{*}\end{array}$} & $55(25.7 \%)$ & 1.00 & \multirow{3}{*}{$\begin{array}{l}0.401 \\
0.109\end{array}$} \\
\hline & $\mathrm{G} / \mathrm{A}$ & $190(49.5 \%)$ & $58(48.3 \%)$ & $0.52(0.27-1.01)$ & & $112(52.3 \%)$ & $0.79(0.46-1.36)$ & \\
\hline & $\mathrm{A} / \mathrm{A}$ & $92(24.0 \%)$ & $27(22.5 \%)$ & $0.42(0.19-0.91)$ & & $47(22.2 \%)$ & $0.60(0.32-1.12)$ & \\
\hline \multirow[t]{2}{*}{ Dominant } & $\mathrm{G} / \mathrm{G}$ & $102(26.6 \%)$ & $35(29.2 \%)$ & 1.00 & \multirow{2}{*}{$0.023^{*}$} & $55(25.7 \%)$ & 1.00 & \multirow{2}{*}{0.219} \\
\hline & G/A-A/A & $282(73.4 \%)$ & $85(70.8 \%)$ & $0.49(0.26-0.91)$ & & $159(74.3 \%)$ & $0.72(0.43-1.21)$ & \\
\hline \multirow[t]{2}{*}{ Recessive } & $\mathrm{G} / \mathrm{G}-\mathrm{G} / \mathrm{A}$ & $292(76.0 \%)$ & $93(77.5 \%)$ & 1.00 & \multirow{2}{*}{0.177} & $167(78.0 \%)$ & 1.00 & \multirow{2}{*}{0.170} \\
\hline & $\mathrm{A} / \mathrm{A}$ & $92(24.0 \%)$ & $27(22.5 \%)$ & $0.64(0.34-1.22)$ & & $47(22.0 \%)$ & $0.70(0.42-1.16)$ & \\
\hline Log-additive & - & - & - & $0.64(0.43-0.95)$ & $0.027^{*}$ & - & $0.77(0.56-1.06)$ & 0.108 \\
\hline
\end{tabular}

OR, odds ratio; 95\% CI, 95\% confidence interval. $p$ values were calculated by unconditional logistic regression analysis with adjustments for age and gender; ${ }^{*} p<0.05$ indicates statistical significance.

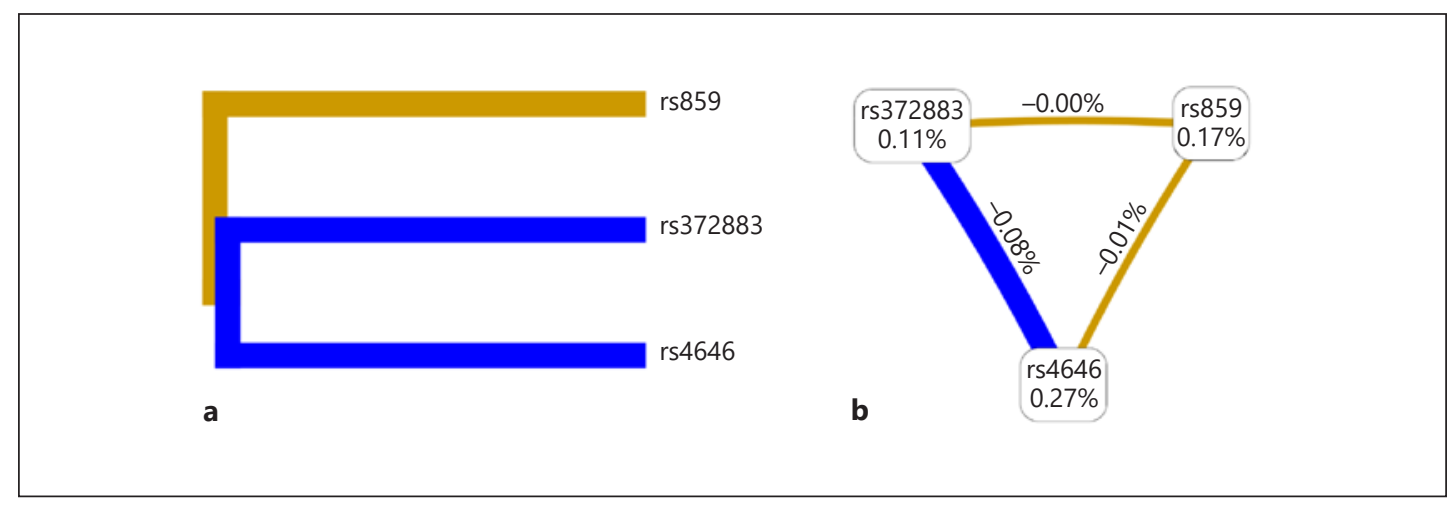

Fig. 1. SNP-SNP interaction dendrogram (a) and Fruchterman-Reingold (b). Yellow and blue represent redundancy or correlation.

We next analyzed whether the genotypic effects on IgAN risk were dependent on gender, two SNPs (rs4646 and rs372883) were found to be significantly associated with IgAN. CYP19A1 rs4646 exhibited a male gender-dependent association with IgAN risk (codominant, $\mathrm{OR}=2.79,95 \% \mathrm{CI}=1.13-6.89, p=0.044$; recessive, $\mathrm{OR}=2.96,95 \% \mathrm{CI}=1.22-7.14, p=0.015$, respectively). BACH1 rs372883 was associated with an increased risk in males (OR $=1.81$, $95 \% \mathrm{CI}=1.03-3.19, p=0.038)$ but with a decreased risk in females (OR $=0.44,95 \% \mathrm{CI}=$ $0.18-0.98, p=0.042$ ) (Table 4).

We also assessed the association between the polymorphisms and susceptibility of IgAN stratified by Lee's classification. As shown in Table 5, subjects with the rare alleles of rs859 showed a significant decreased IgAN risk in the grade I-II subgroup in genotype model (OR = $0.42,95 \% \mathrm{CI}=0.19-0.91, p=0.028)$, dominant model $(\mathrm{OR}=0.49,95 \% \mathrm{CI}=0.26-0.91, p=$ 0.023 ), and additive model (OR $=0.64,95 \% \mathrm{CI}=0.43-0.95, p=0.027$ ).

Subsequently, MDR analysis was implemented to assess the impact of the SNP-SNP interactions. As was shown in Figure 1, the dendrogram and the Fruchterman-Reingold described 
Yang et al.: Genetic Variations rs859, rs4646, and rs372883 Are Associated with IgA Nephropathy Risk

Table 6. SNP-SNP interaction models analyzed by the MDR method

\begin{tabular}{llllll}
\hline Model & $\begin{array}{l}\text { Training } \\
\text { Bal. Acc. }\end{array}$ & $\begin{array}{l}\text { Testing } \\
\text { Bal. Acc. }\end{array}$ & CVC & OR (95\% CI) & $p$ \\
\hline rs372883 (BACH1) & 0.519 & 0.451 & $7 / 10$ & $1.29(0.95-1.76)$ & 0.098 \\
rs4646 (CYP19A1), rs859 (IL16) & 0.541 & 0.471 & $7 / 10$ & $1.59(1.13-5.25)$ & $0.008^{*}$ \\
$\begin{array}{l}\text { rs372883 (BACH1), rs4646 } \\
\quad \text { (CYP19A1), rs859 (IL16) }\end{array}$ & 0.566 & 0.487 & $10 / 10$ & $1.87(1.36-2.57)$ & $<0.001^{*}$ \\
\hline
\end{tabular}

MDR, multifactor dimensionality reduction; Bal. Acc., balanced accuracy; CVC, cross-validation consistency; OR, odds ratio; 95\% CI, 95\% confidence interval. $p$ values were calculated using $\chi^{2}$ tests. ${ }^{*} p<0.05$ indicates statistical significance.

the interactions between these SNPs. Table 6 showed that rs372883 was the best single-locus model to predict IgAN (testing accuracy $=0.451, \mathrm{CVC}=7 / 10, p=0.0978$ ). The best two-locus model was the combination of rs372883 and rs4646 (testing accuracy $=0.471, \mathrm{CVC}=7 / 10$, $p=0.0082$. The three-locus model included rs372883, rs4646 and rs859 (testing accuracy $=$ $0.487, \mathrm{CVC}=10 / 10, p=0.0001$ ).

Finally, the possible relationships between rs859, rs4646 and rs372883 polymorphisms and clinical variables in patients were investigated. IgAN patients were classified into three groups according to the three genotypes, and the means of patient quantitative traits were calculated. The results showed that no significant associations were identified between rs859 and rs372883 genotype polymorphisms and all of the above-mentioned clinical parameters ( $p>0.05$, shown in Table 7). Interestingly, the CA genotype $(173.34 \pm 213.07 \mu \mathrm{mol} / \mathrm{L})$ of rs4646 were higher serum creatinine (SCr) level than CC genotype $(129.15 \pm 108.06 \mu \mathrm{mol} / \mathrm{L})$ and AA genotype $(121.49 \pm 117.08 \mu \mathrm{mol} / \mathrm{L})(p=0.035)$, but not with other above-mentioned clinical parameters.

\section{Discussion}

IgAN is considered to be a polygenic and multifactorial disorder. The etiology of IgAN is poorly understood, but genetic and environmental factors are thought to play important roles in this disease. Previous studies have reported that multiple gene polymorphisms are correlated with the susceptibility to IgAN, including CCDC132, FDX1, TNFSF13 and IFN- $\gamma$ gene variants $[13,14]$. SNPs on regulatory regions $\left(3^{\prime}\right.$ - and $5^{\prime}$-UTR), miRNA-binding sites, and exon variants of the genes have been the center of attention in the last few years, but are few reported in IgAN.

We performed a case-control study to investigate the association between polymorphisms in the $3^{\prime}$-UTR of the target genes and the susceptibility to IgAN. The result revealed that only $I L-16$ rs859 and CYP19A1 rs4646 variants were associated with the susceptibility to IgAN in the overall. Interestingly, our further stratified analysis showed that CYP19A1 rs4646 and $B A C H 1$ rs372883 increased the risk of IgAN in males while BACH1 rs372883 decreased IgAN susceptibility in females. We also found that rs859 polymorphism was significantly associated with Lee's grade of IgAN. Given that IgAN represents a complex disorder influenced by the interplay between genetic and environmental factors, multi-gene or SNP-SNP interaction studies may help discover the risk factors for IgAN. Accordingly, we performed the MDR to determine the potential SNP-SNP interactions among $I L-16$ rs859, CYP19A1 rs4646 and $B A C H 1$ rs372883. The analysis of the SNP-SNP interactions showed a strong interaction 
Yang et al.: Genetic Variations rs859, rs4646, and rs372883 Are Associated with IgA Nephropathy Risk

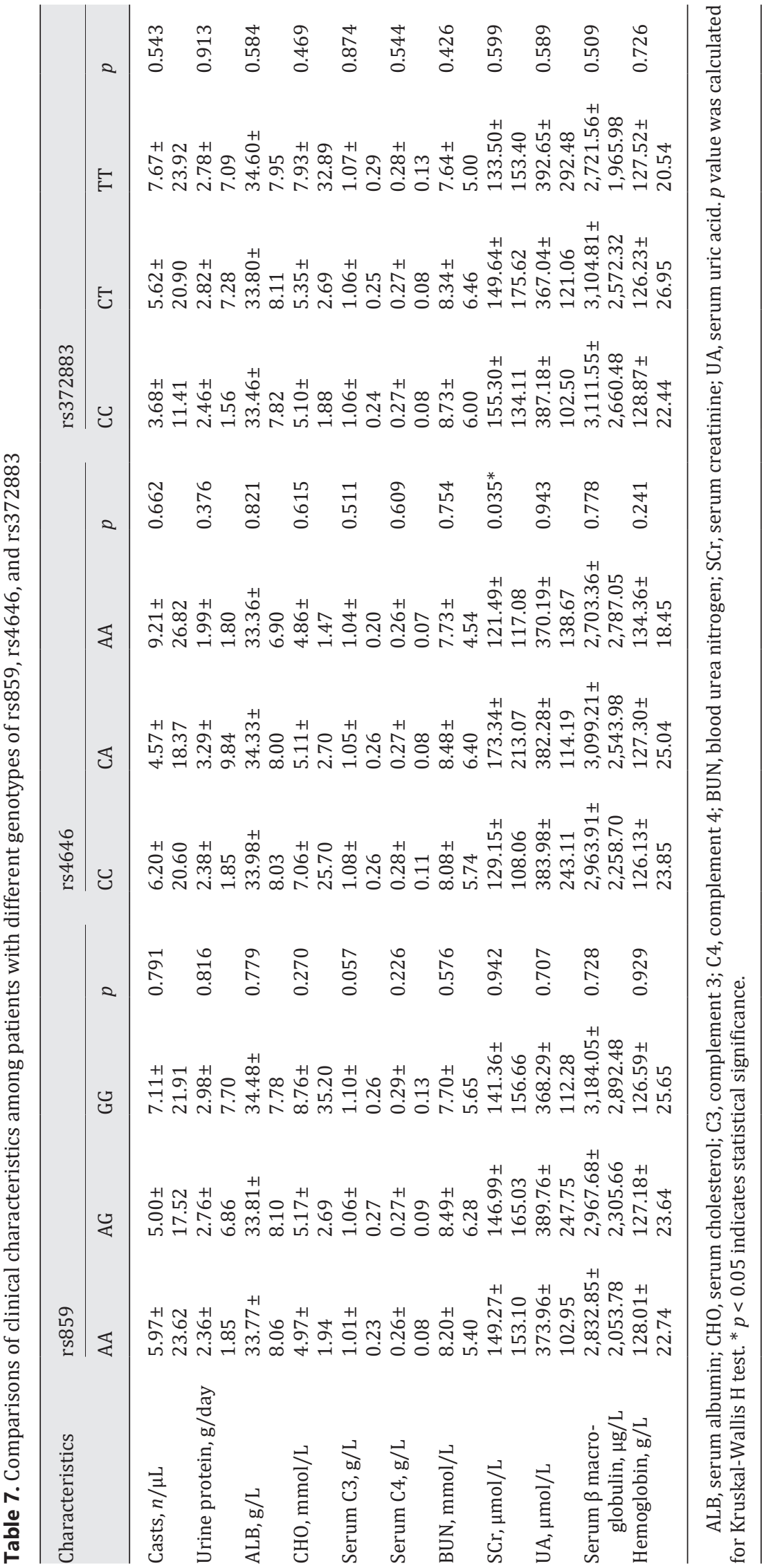


Yang et al.: Genetic Variations rs859, rs4646, and rs372883 Are Associated with IgA Nephropathy Risk

between rs859, rs4646, and rs372883 regarding susceptibility to IgAN. To the best of our knowledge, this is the first study demonstrating the effects of the relationships between these 3 '-UTR SNPs and the risk of IgAN.

Interleukin-16 (IL-16) located on chromosome 15. q26.3, as a multifunctional cytokine that involved in the chemoattraction and modulation of $\mathrm{T}$ cell activation, is an important mediator of inflammatory and autoimmune diseases. Previous studies have reported that genetic variations of $I L-16$ are associated with susceptibility to various renal diseases, such as end-stage renal failure and renal cell carcinoma $[15,16]$. Rs859 $(\mathrm{G}>\mathrm{A})$ polymorphism in $3^{\prime}$-UTR of $I L-16$ mRNA is located in miR-188-5p and miR-361-3p binding sites or adjacent region based on database prediction. Previous miRNA profile analysis revealed that miR188-5p and miR-361-3p were frequently overexpressed in peripheral-blood mononuclear cells of patients with IgAN [17]. Our analysis revealed that rs859 polymorphism was similar to reduce the risk of IgAN ( $p=0.04$; adjusted OR $=0.75$; 95\% CI: $0.57-0.99)$. Therefore, one hypothesis potentially explaining $I L-16$ rs859 to the susceptibility of IgAN is that genetic variation may affect the binding of miR-188-5p and miR-361-3p to the $I L-16$ mRNA. However, further study is necessary to confirm this hypothesis.

Cytochrome P450 (CYP) metabolites of arachidonic acid, 20-hydroxyeicosatetraenoic acid and epoxyeicosatrienoic acids play an important role for the regulation of renal tubular and vascular function $[18,19]$. CYP19A1 is part of a cluster of cytochrome P450 genes on chromosome 15q21.1, which is responsible for the biosynthesis of androgens and estrogen [20]. Some animal experiments and clinical observations have demonstrated that the occurrence of kidney disease is related to the levels of sex hormones throughout the body [21, 22] Therefore, it is not surprising that CYP19A1 is associated with IgAN. Many polymorphisms have been identified with the CYP19A1 gene, which have been associated with altered sex hormone levels in serum and urine [23, 24]. Rs4646 polymorphisms in the $3^{\prime}$-UTR of CYP19A1 could reduce levels of circulating sex hormone [25] and alter protein activation. We found that patient with rs4646 AA genotype have a statistically significant association with 2.38fold increased susceptibility to IgAN compared with CC genotype. As known, IgAN is more common in males than in females. Gender stratification results showed that rs 4646 polymorphisms contributed to the susceptibility to IgAN in males ( $p=0.044$, adjusted OR $=2.79,95 \%$ CI: 1.13-6.89). Let-7b and let-7d were overexpression in patients with IgAN [17], and could bind with the 3 '-UTR of CYP19A1 on computational data. It is biologically plausible that the 3 '-UTR CYP19A1 variation perhaps changes the biological activities by altering transcription and translation of CYP19A1 gene, thus inducing the higher risk of IgAN.

$B A C H 1$ (BTB and CNC homology 1) located at chromosome 21q21.3, which is recognized as a recently hypoxia regulator and functions as a transcription regulator of the heme oxygenase (HO)- 1 and $\beta$-globin genes in many human cell types in response to oxidative stress [26]. It has been clinically demonstrated that the immunoreactivities of intrarenal HO-1 in IgAN patients were significantly increased as compared to those of control subjects [27]. BACH1 was identified to be a target of miR-155 in renal cancer [28]. Furthermore, Wang et al. reported that intra-renal and urinary levels of miR-155 were significantly elevated in IgAN, and the degree of upregulation was correlated with clinical and histological severity as the disease [29]. BACH1 gene is regulated by miR-155 through the predicted binding site in the $3^{\prime}$-UTR of BACH1 in silico. In the present study, we analyzed rs372883 $\mathrm{T}>\mathrm{C}$ polymorphism within the miR-155 seed region at the 3'-UTR of BACH1 in IgAN. There was no association of rs372883 polymorphism with IgAN risk in the overall. Interestingly, our results showed that CC genotype significantly increased the risk of IgAN in males, but CT-CC genotype was related with the reduced IgAN susceptibility among females. As noted previously, IgAN is more common in males than in females. This led us to hypothesize that rs $372883 \mathrm{C}$ allele might be associated with the etiology of IgAN, especially in males. 


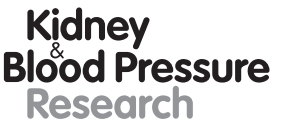

Research

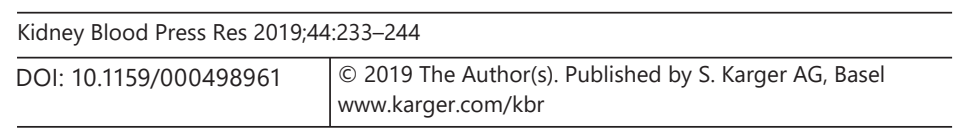

Yang et al.: Genetic Variations rs859, rs4646, and rs372883 Are Associated with IgA Nephropathy Risk

Several intrinsic limitations to our study should be considered. First, the selection bias in this case-control study was its hospital-based design, and therefore it may not be representative of the general population. Second, environmental exposure was not available, which limited us to further analyze the potential interaction of gene-environment on IgAN risk. Third, the SNPs were located in the 3'-UTR region of the target genes, which binding with miRNA was identified based on bioinformatics software. Further functional assay is necessary to reveal their relevance. Despite the limitations mentioned above, the results of our present study provided scientific evidence about $I L-16, C Y P 19 A 1$ and BACH1 genes with IgAN in the future studies.

\section{Conclusion}

In summary, our results provided the first evidence that $I L-16$ rs859, CYP19A1 rs4646 and $B A C H 1$ rs372883 variants were associated with risk of IgAN in Chinese Han population, which may shed light on the new candidate genes and novel ideas for the study of the subsequent occurrence mechanisms of IgAN.

\section{Acknowledgements}

We are grateful to the individuals for their participation in this study. We also thank the clinicians and hospital staff who contributed to the sample and data collection for this study. We would also like to thank all participants for this manuscript.

\section{Disclosure Statement}

The authors declare no conflict of interest.

\section{Funding Sources}

This work is supported by National Natural Science Foundation of China (No. 81560124), and Hainan key research and development projects (ZDYF2018131, ZDYF2017113 and ZDYF2017114) and Hainan science and technology planned project of Youth outstanding ability of innovation (201704).

\section{References}

1 Wyatt RJ, Julian BA. IgA nephropathy. N Engl J Med. 2013 Jun;368(25):2402-14.

2 Maisonneuve P, Agodoa L, Gellert R, Stewart JH, Buccianti G, Lowenfels AB, et al. Distribution of primary renal diseases leading to end-stage renal failure in the United States, Europe, and Australia/New Zealand: results from an international comparative study. Am J Kidney Dis. 2000 Jan;35(1):157-65.

3 Suzuki K, Honda K, Tanabe K, Toma H, Nihei H, Yamaguchi Y. Incidence of latent mesangial IgA deposition in renal allograft donors in Japan. Kidney Int. 2003 Jun;63(6):2286-94.

4 Feehally J, Barratt J. The Genetics of IgA Nephropathy: An Overview from Western Countries. Kidney Dis (Basel). 2015 May;1(1):33-41.

5 Oortwijn BD, van der Boog PJ, Roos A, van der Geest RN, de Fijter JW, Daha MR, et al. A pathogenic role for secretory IgA in IgA nephropathy. Kidney Int. 2006 Apr;69(7):1131-8. 


\section{Kidney \\ Blood Pressure \\ Research}

\begin{tabular}{l|l}
\hline Kidney Blood Press Res 2019;44:233-244 \\
\hline DOI: 10.1159/000498961 & $\begin{array}{l}\text { @ 2019 The Author(s). Published by S. Karger AG, Basel } \\
\text { www.karger.com/kbr }\end{array}$ \\
\hline
\end{tabular}

Yang et al.: Genetic Variations rs859, rs4646, and rs372883 Are Associated with IgA Nephropathy Risk

6 Suzuki Y, Matsuzaki K, Suzuki H, Okazaki K, Yanagawa H, Ieiri N, et al. Serum levels of galactose-deficient immunoglobulin (Ig) A1 and related immune complex are associated with disease activity of IgA nephropathy. Clin Exp Nephrol. 2014 Oct;18(5):770-7.

7 Li PK, Ho KK, Szeto CC, Yu L, Lai FM. Prognostic indicators of IgA nephropathy in the Chinese-clinical and pathological perspectives. Nephrol Dial Transplant. 2002 Jan;17(1):64-9.

8 Zhang C, Zeng X, Li Z, Wang Z, Li S. Immunoglobulin A nephropathy: current progress and future directions. Transl Res. 2015 Aug;166(2):134-44.

9 Syvänen AC: Toward genome-wide SNP genotyping. Nat Genet. 2005 Jun;37 Suppl:S5-10.

10 Diamandis M, White NM, Yousef GM. Personalized medicine: marking a new epoch in cancer patient management. Mol Cancer Res. 2010 Sep;8(9):1175-87.

11 Zhou F, He X, Liu H, Zhu Y, Jin T, Chen C, et al. Functional polymorphisms of circadian positive feedback regulation genes and clinical outcome of Chinese patients with resected colorectal cancer. Cancer. 2012 Feb; 118(4):937-46.

12 Kertesz M, Iovino N, Unnerstall U, Gaul U, Segal E. The role of site accessibility in microRNA target recognition. Nat Genet. 2007 Oct;39(10):1278-84.

13 Niu D, Ren Y, Xie L, Sun J, Lu W, Hao Y, et al. Association between CCDC132, FDX1 and TNFSF13 gene polymorphisms and the risk of IgA nephropathy. Nephrology (Carlton). 2015 Dec;20(12):908-15.

14 Gao J, Wei L, Liu X, Wang L, Niu D, Jin T, et al. Association Between IFN- $\gamma$ Gene Polymorphisms and IgA Nephropathy in a Chinese Han Population. Kidney Blood Press Res. 2017;42(1):136-44.

15 Zhao J, Bolton EM, Randle L, Bradley JA, Lever AM. Functional characterization of late outgrowth endothelial progenitor cells in patients with end-stage renal failure. Transpl Int. 2014 May;27(5):437-51.

16 Obara W. Editorial comment to IL-16 polymorphism and risk of renal cell carcinoma: association in a Chinese population. Int J Urol. 2010 Aug;17(8):707.

17 Serino G, Sallustio F, Cox SN, Pesce F, Schena FP. Abnormal miR-148b expression promotes aberrant glycosylation of IgA1 in IgA nephropathy. J Am Soc Nephrol. 2012 May;23(5):814-24.

18 Capdevila JH, Falck JR, Harris RC. Cytochrome P450 and arachidonic acid bioactivation. Molecular and functional properties of the arachidonate monooxygenase. J Lipid Res. 2000 Feb;41(2):163-81.

19 Eid S, Maalouf R, Jaffa AA, Nassif J, Hamdy A, Rashid A, et al. 20-HETE and EETs in diabetic nephropathy: a novel mechanistic pathway. PLoS One. 2013 Aug;8(8):e70029.

20 Numazawa M, Handa W, Hasegawa C, Takahashi M. Structure-activity relationships of 2alpha-substituted androstenedione analogs as aromatase inhibitors and their aromatization reactions. J Steroid Biochem Mol Biol. 2005 Dec;97(4):353-9.

21 Tang X, Gu R, Xie XS, Wu T. Androgens for the anaemia of chronic kidney disease in adults. John Wiley \& Sons, Ltd; 2008. https://doi.org/10.1002/14651858.CD006881.

22 Naghii MR, Mofid M, Hedayati M, Khalagi K. Antioxidants inhibition of high plasma androgenic markers in the pathogenesis of ethylene glycol (EG)-induced nephrolithiasis in Wistar rats. Urolithiasis. 2014 Apr;42(2): 97-103.

23 Huber JC, Schneeberger C, Tempfer CB. Genetic modelling of the estrogen metabolism as a risk factor of hormone-dependent disorders. Maturitas. 2002 May;42(1):1-12.

24 Tworoger SS, Chubak J, Aiello EJ, Ulrich CM, Atkinson C, Potter JD, et al. Association of CYP17, CYP19, CYP1B1, and COMT polymorphisms with serum and urinary sex hormone concentrations in postmenopausal women. Cancer Epidemiol Biomarkers Prev. 2004 Jan;13(1):94-101.

25 Haiman CA, Dossus L, Setiawan VW, Stram DO, Dunning AM, Thomas G, et al. Genetic variation at the CYP19A1 locus predicts circulating estrogen levels but not breast cancer risk in postmenopausal women. Cancer Res. 2007 Mar;67(5):1893-7.

26 Sun J, Hoshino H, Takaku K, Nakajima O, Muto A, Suzuki H, et al. Hemoprotein Bach1 regulates enhancer availability of heme oxygenase-1 gene. EMBO J. 2002 Oct;21(19):5216-24.

27 Ohashi N, Urushihara M, Kobori H: Activated intrarenal reactive oxygen species and renin angiotensin system in IgA nephropathy. Clin Exp Pharmacol Physiol. 2010;36(8):750-5.

28 Li S, Chen T, Zhong Z, Wang Y, Li Y, Zhao X. microRNA-155 silencing inhibits proliferation and migration and induces apoptosis by upregulating BACH1 in renal cancer cells. Mol Med Rep. 2012 Apr;5(4):949-54.

29 Wang G, Kwan BC, Lai FM, Chow KM, Li PK, Szeto CC. Elevated levels of miR-146a and miR-155 in kidney biopsy and urine from patients with IgA nephropathy. Dis Markers. 2011;30(4):171-9. 